\title{
Control of magnetic properties in metallo-organic thin films
}

\author{
Thomas Gredig • K. Paul Gentry • \\ Corneliu N. Colesniuc • Ivan K. Schuller
}

Received: 29 November 2009/Accepted: 11 February 2010/Published online: 24 February 2010

(C) The Author(s) 2010. This article is published with open access at Springerlink.com

\begin{abstract}
Magnetic metallo-organic thin films have been prepared at different growth temperatures to characterize the influence of structural properties on the magnetic characteristics. A quantitative analysis of the grain size shows that the growth temperature increases the elliptical grain size considerably. Long grains of planar iron phthalocyanine form quasi one-dimensional iron chains, which are embedded in a carbon matrix along the other two dimensions. The chains are narrowly spaced $(1.3 \mathrm{~nm})$ and run parallel to the substrate. Temperature-dependent magnetization curves depend on the structural details of the iron chains. These magnetic properties are tuned with deposition parameters.
\end{abstract}

\section{Introduction}

Low-dimensional magnetism has played an important role in theoretical physics, including the famous model of Ising chains [1]. Experimentally however, magnetic monatomic metal chains are challenging to fabricate and analyze [2,3]. Many theoretical models for molecular-based magnets with low-dimensional magnetic structures yield interesting results, but suitable experimental systems mostly lack $[4,5]$. In the past, iron stripes have been prepared with an average line separation of $3-10 \mathrm{~nm}$ using terraced

T. Gredig $(\bowtie) \cdot$ K. P. Gentry

Department of Physics and Astronomy, California State

University Long Beach, 1250 Bellflower Blvd., Long Beach,

CA 90840, USA

e-mail: tgredig@csulb.edu

C. N. Colesniuc · I. K. Schuller

Department of Physics and Astronomy, University of California

San Diego, 9500 Gilman Dr., La Jolla, CA 92093, USA substrates. A significant reduction of the line separation is achieved with metallo-organic molecules. These molecules have several advantages such as control of size, ease of self-assemblage, and chemical tunability. Here, the magnetic properties of iron phthalocyanine $(\mathrm{FePc})$ thin films are explored. Phthalocyanines are planar molecules with a metallic atom at the core [6]. Thin films can be prepared, such that the plane of the molecule is perpendicular to the substrate plane. Then, the $b$-axis of the molecule's unit cell runs parallel to the substrate plane as shown in Fig. 1. In this way, metallic one-dimensional chains are formed, where the length of the chain is limited by the crystallite size in the thin film. The surface energy produces the preferential growth along the $b$-axis following a herringbone structure. In the following, the deposition temperature is used as an experimental tuning parameter of the crystallite size. The structural effects on the magnetic properties of iron chains are then discussed.

\section{Experiment}

Metallo-organic thin films of $\mathrm{FePc}$ were deposited onto carefully cleaned sapphire substrates [7, 8]. FePc was purchased from Sigma-Aldrich. Subsequently, the powder was purified three times using the temperature gradient sublimation method in vacuum $\left(<10^{-6}\right.$ Torr) [9]. The FePc was deposited using an effusion cell in an ultra-high vacuum chamber with the base pressure of $5 \times 10^{-9}$ Torr. The substrate temperature during the deposition was varied from room temperature to $260{ }^{\circ} \mathrm{C}$ to increase diffusion on the surface [10]. As the thickness of the thin film increases during growth, new grains may form on top of the first layer of grains. Such grains would overlap and partially cover grains in a lower level. Therefore, the layer thickness 
(a)

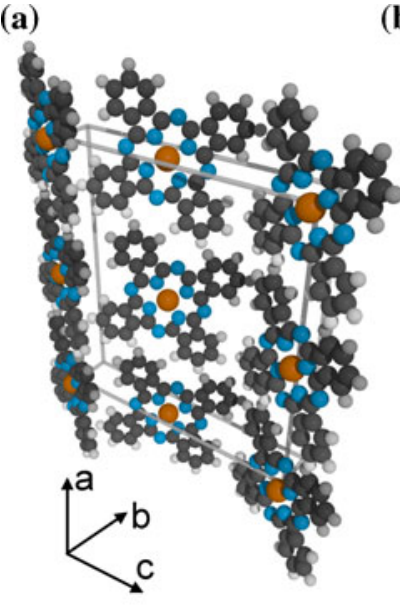

(b)

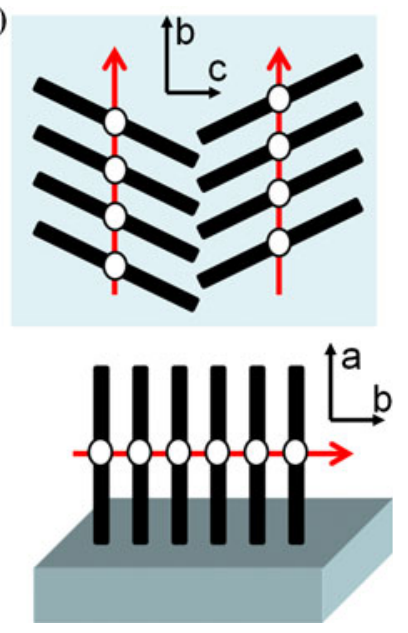

Fig. 1 a The unit cell of iron phthalocyanine $\left(\mathrm{FeC}_{32} \mathrm{~N}_{8} \mathrm{H}_{16}\right)$ is shown for the $\alpha$-phase. The lattice parameters are $a=25.9 \AA, b=3.8 \AA$, $c=24.1 \AA$, and $\beta=90^{\circ}$. The unit cell contains four atoms. For clarity, six molecules in the back plane of the unit cell are omitted. b A schematic top-view of two parallel iron chains displays the herringbone structure (top). The chains run parallel to the sapphire surface as seen in the side-view (bottom). It should be noted that in the side-view the molecule is tilted with respect to the $b$-axis, implying that the iron-iron link is not the shortest distance between adjacent molecules. In fact, the tilt angle of the $\alpha$-phase in FePc implies that the iron-nitrogen link is the closest

needs to be thin enough to prevent the second layer to establish in order to measure grain sizes reliably and quantitatively [10]. All films are 20 monolayers $(27 \mathrm{~nm})$ thick, which is smaller than the smallest average grain size for all samples $(31 \mathrm{~nm})$, while providing enough volume for magnetic measurements with a vibrating sample magnetometer (VSM). The sample thickness is inferred from a calibrated sample that has been measured with $x$-ray diffraction. The surface morphology of the sample was measured with atomic force microscopy (AFM) using a NanoScope III multimode.

\section{Analysis}

The growth of FePc molecules on heated sapphire surfaces is a complex nonequilibrium process involving adsorption

and diffusion at ambient temperatures. At higher temperatures, re-desorption from the surface needs to be taken into account. As a result of this process rounded grains are formed at low-deposition temperatures $\left(<80{ }^{\circ} \mathrm{C}\right)$ and they become elongated at intermediate temperatures. At high temperatures $\left(>200{ }^{\circ} \mathrm{C}\right)$, phthalocyanine undergoes a phase transition from a metastable $\alpha$ - to a stable $\beta$-phase, which results in rougher surfaces with very long grains (see Fig. 2c) [11]. An Arrhenius law fit in the temperature range below $230{ }^{\circ} \mathrm{C}$ yields an activation energy of $87 \mathrm{meV}$ as shown in Fig. 3. This is slightly lower than the activation energy found in other van-der-Waals dominated molecules, such as sexithiophene thin films [12].

On sapphire, FePc thin films grow with the $b$-axis parallel to the substrate as determined from $\mathrm{X}$-ray diffraction [6]. Thus, the chains form parallel to the substrate plane (see Fig. 1). However, the direction of the chains is randomly distributed in the plane of the film. The grains can be approximated as ellipses where the major axis corresponds to the length of the one-dimensional iron chains.

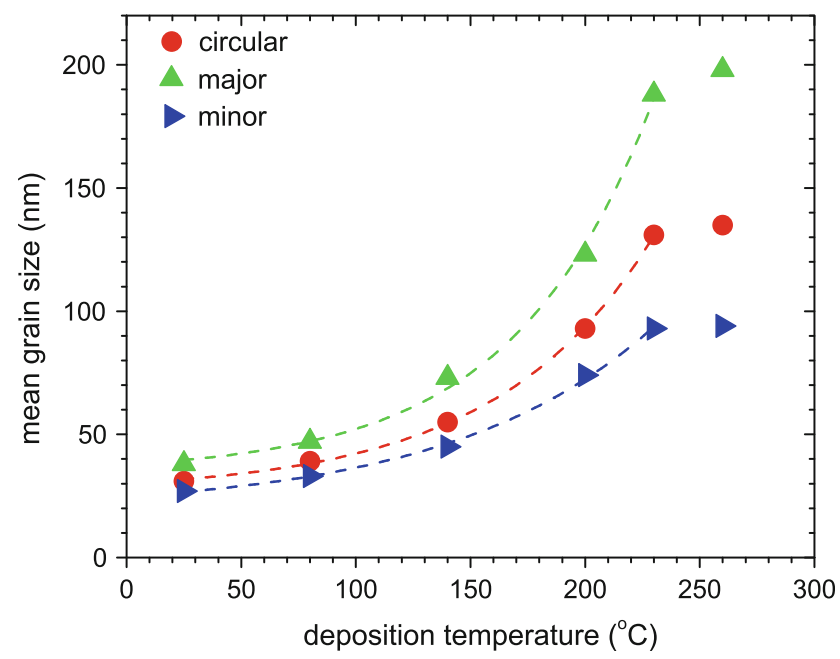

Fig. 3 Mean grain size of FePc thin films deposited at different temperatures; all films are $27 \mathrm{~nm}$ thick and were grown on sapphire substrates. Grain sizes are fit to ellipses and mean major and minor axis lengths are determined from AFM images. Lines are exponential fits to the data excluding the highest temperature, which corresponds to a different FePc phase
Fig. 2 The AFM images show the surface morphology of three 27nm-thick FePc thin films deposited at a $80{ }^{\circ} \mathrm{C}$, b $200{ }^{\circ} \mathrm{C}$, and $\mathbf{c} 230^{\circ} \mathrm{C}$. The $b$-axis is parallel to the substrate plane and points along the elongation axis of the grains

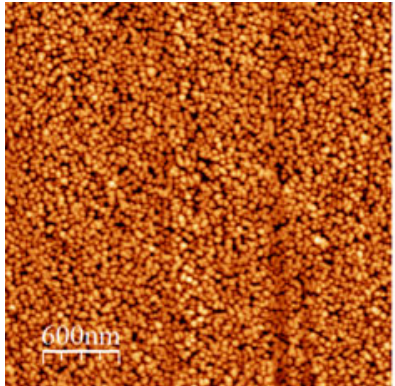

(a)

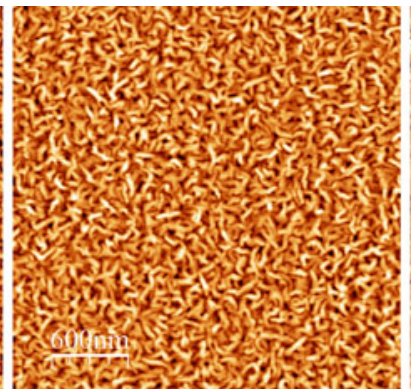

(b)

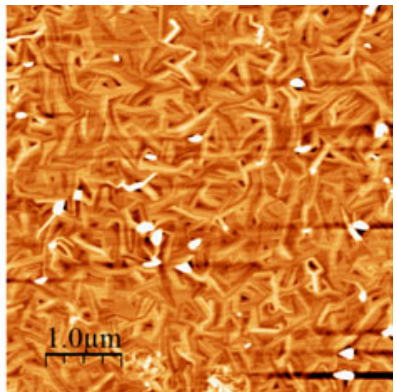

(c) 
Chains are separated both horizontally and vertically by a carbon matrix such that the iron interchain distances are $1.3 \mathrm{~nm}$ or about four times larger than the iron intrachain distance, which is $0.34 \mathrm{~nm}$ [13]. The grain size can be inferred from AFM images. In this analysis, each grain is fit to an ellipse using a watershed algorithm to extract the minor and major axis component [10]. Consequently, the small rounded grains consist of chains with $100 \mathrm{Fe}$ atoms, while the high temperature deposited samples have average chains that are $600 \mathrm{Fe}$ atoms long. Additionally, each grain consists of many chains that are parallel to each other. In the case of the $25^{\circ} \mathrm{C}$ sample, there are about 30 parallel chains in width and 20 parallel chains in height.

The control of the average chain length is used to study the magnetic properties of these quasi one-dimensional magnetic chains. The applied field in the VSM is parallel to the substrate plane. FePc shows hysteretic magnetization behavior below $4.5 \mathrm{~K}$ (Fig. 4). A coercive field on the order of $300 \mathrm{G}$ is recorded. Additionally, the curves are pinched distinctively and show two characteristic pockets in the regions between 500 and $3500 \mathrm{G}$ similar to observations in powder specimens [13]. The samples behave paramagnetically at or above $4.5 \mathrm{~K}$ and show no hysteresis. Evangelisti et al. proposed that magnetic Fe ions interact ferromagnetically along the chain direction, but individual chains couple antiferromagnetically with the chain moment allowed to cant, because the magnetic moment of the $\mathrm{Fe}$ ion tends to point perpendicular to the molecular plane.

The magnetic properties of samples with different iron chain lengths are compared with magnetization versus temperature curves. After applying $1 \mathrm{~T}$, the sample's magnetization is measured in-field $(500 \mathrm{G})$ during sample

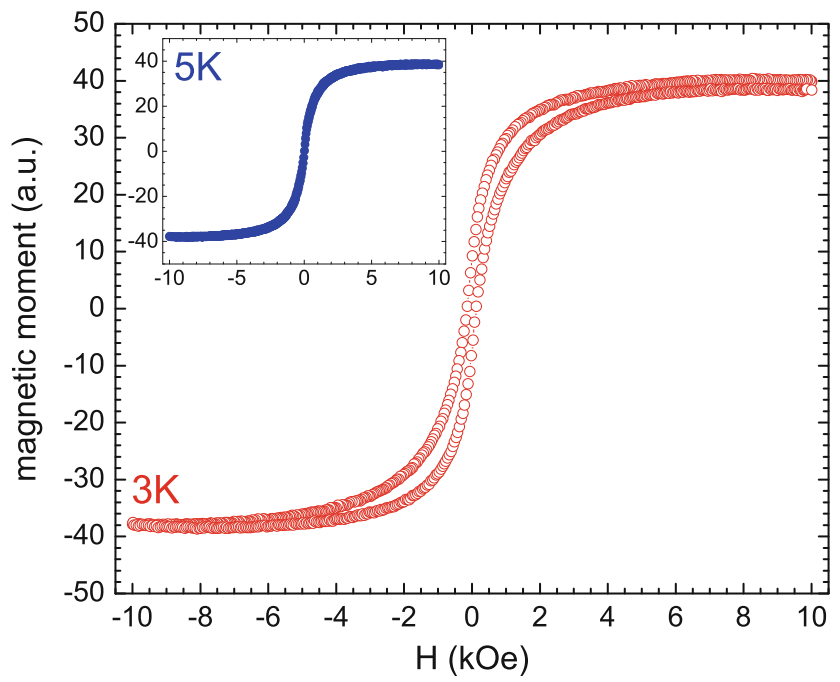

Fig. 4 Hysteresis loops measured in FePc sample deposited at $80{ }^{\circ} \mathrm{C}$. The hysteretic effects present at $3 \mathrm{~K}$ are absent at $5 \mathrm{~K}$ (inset) warming. After subtracting the diamagnetic background moment of the sapphire substrate, the magnetic moment per volume of the samples varies between 1.5 and 6 emu/ $\mathrm{cm}^{3}$. Since the total magnetic moment produced by the FePc thin film is small compared to the larger diamagnetic background, the residual background is offset using the value at a chosen temperature $(100 \mathrm{~K})$, which is well above the critical temperature in FePc. The resulting curves overlap within noise of the measurements in the temperature range between 40 and $300 \mathrm{~K}$, but diverge at low temperatures as shown Fig. 5.

The magnetic moment is bigger for samples deposited at higher substrate temperatures which have a larger crystallite size. This result is shown in the inset of Fig. 5, where the magnetic moment measured at $500 \mathrm{G}$ and $5 \mathrm{~K}$ is plotted as a function of the substrate temperature. The signal is normalized to the sample size. Since the substrate temperature and associated change in chain length have no markable effect on the coercive fields, but rather affect the magnitude of the magnetic moment, then the role of the slight lattice spacing contractions that occur with higher substrate temperature may be most important [7]. The van der Waals interactions between individual FePc molecules allow small lattice changes to occur with higher deposition temperature. Thus, the lattice spacing may prompt the moment increase observed in Fig. 5. Further evidence is needed to conclusively determine the origin of the magnetic moment increase seen in Fig. 5. One possibility would be experiments with fluorinated FePc for which the intrachain distance is $7 \%$ larger compared to FePc.

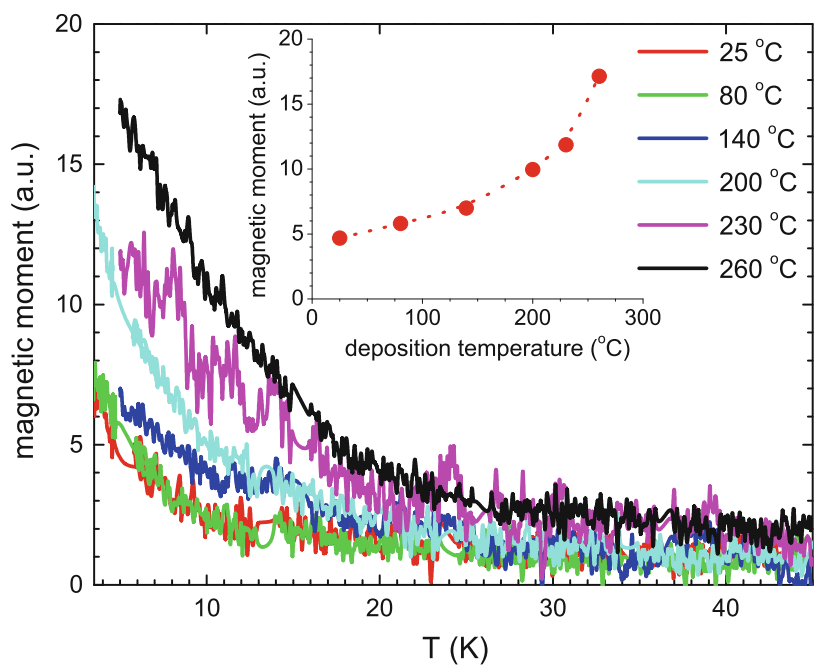

Fig. 5 The magnetic moment of FePc thin films is measured as the samples are warmed in a constant magnetic field of $500 \mathrm{G}$. The samples were prepared at different deposition temperatures ranging from 25 to $260{ }^{\circ} \mathrm{C}$. Inset: The magnetic moment at $5 \mathrm{~K}$ measured in $500 \mathrm{G}$ increases with the substrate deposition temperature 


\section{Conclusion}

Iron phthalocyanine thin films are studied to explore lowdimensional magnetism in closely coupled monatomic metallic chains. The chain separation is very uniform and measures $1.3 \mathrm{~nm}$. The substrate temperature during deposition allows direct control of the iron chain length. Samples have average chain lengths varying from $38 \mathrm{~nm}$ to almost $200 \mathrm{~nm}$. At sufficiently low temperatures, hysteresis in magnetization curves is observed for all samples. The chain length has no measurable effect on the coercivity or critical temperature. However, temperature-dependent magnetization curves show an increased moment for samples deposited at higher temperatures. The increased moment may be correlated with a lattice contraction occurring with higher deposition temperatures.

Acknowledgements This research was supported by NSF grant DMR-0847552, a grant by AFOSR, by the UCOP program on Carbon-based Nanostructures, and California State University, Long Beach. The equipment for the magnetic measurements was purchased under the NSF MRI-0619909 grant. One of the authors (T.G.) would like to thank Prof. Jiyeong Gu for help with VSM measurements.

Open Access This article is distributed under the terms of the Creative Commons Attribution Noncommercial License which permits any noncommercial use, distribution, and reproduction in any medium, provided the original author(s) and source are credited.

\section{References}

1. Thouless DJ (1969) Phys Rev 187(2):732

2. Shen J, Skomski R, Klaua M, Jenniches H, Manoharan SS, Kirschner J (1997) Phys Rev B 56(5):2340

3. Gambardella P, Blanc M, Brune H, Kuhnke K, Kern K (2000) Phys Rev B 61(3):2254

4. Emberly EG, Kirczenow G (2002) Chem Phys 281(2-3):311

5. Yao K, Fu H, Liu Z (2005) Solid State Commun 135(3):197

6. Liu G, Gredig T, Schuller IK (2008) Europhys Lett 83:56001

7. Miller CW, Sharoni A, Liu G, Colesniuc CN, Fruhberger B, Schuller IK (2005) Phys Rev B 72(10):104113

8. Yang RD, Gredig T, Colesniuc CN, Park J, Schuller IK, Trogler WC, Kummel AC (2007) Appl Phys Lett 90(26):263506

9. Wagner HJ, Loutfy RO, Hsiao C (1982) J Mater Sci 17(10): 2781. doi:10.1007/BF00644652

10. Gentry KP, Gredig T, Schuller IK (2009) Phys Rev B 80(17): 174118. doi:10.1103/PhysRevB.80.174118

11. Heutz S, Bayliss S, Middleton RL, Rumbles G, Jones TS (2000) J Phys Chem B 104(30):7124

12. Biscarini F, Samorí P, Greco O, Zamboni R (1997) Phys Rev Lett 78(12):2389

13. Evangelisti M, Bartolomé J, de Jongh LJ, Filoti G (2002) Phys Rev B 66(14):144410 